\title{
Roles of Circular RNAs in Neurologic Disease
}

\author{
Yiye Shao ${ }^{1,2}$ and Yinghui Chen ${ }^{1,2 *}$ \\ ${ }^{1}$ Department of Neurology, Jinshan Hospital, Fudan University, Shanghai, China, ${ }^{2}$ Department of Neurology, Shanghai \\ Medical College, Fudan University, Shanghai, China
}

Circular RNAs (circRNAs) are a novel type of endogenous noncoding RNA receiving increasing attention. They have been shown to act as a natural microRNA sponges that repress the activity of corresponding miRNAs by binding with them, thus regulating target genes. Numerous studies have shown that miRNAs are involved in the pathogenesis of neurological diseases. Therefore, circRNAs may act as important regulatory factors in the occurrence and development processes of neurological disease.

Keywords: circular RNA, non-coding RNA, neurological disease, microRNA, pathogenesis

Differences between species are embodied in both gene numbers and the regulation of gene expression. Recent studies have demonstrated that non-coding RNAs play an important role in regulating gene expression. CircRNA was first discovered as a novel type of endogenous non-coding RNAs in the 1970s (Sanger et al., 1976; Arnberg et al., 1980; Cocquerelle et al., 1993), however, the function of these special structures has only recently been revealed and appreciated. In this review, we will highlight recent studies of the latest progress in the circRNAs field, in the context of neurological diseases.

OPEN ACCESS

Edited by:

Ildikó Rácz,

University of Bonn, Germany

Reviewed by:

Michele Papa,

Seconda Università di Napoli, Italy Eldi Schonfeld-Dado,

Stanford University, USA

*Correspondence:

Yinghui Chen

yinghuichen@fudan.edu.cn

Received: 13 February 2016 Accepted: 27 March 2016 Published: 13 April 2016

Citation: Shao Y and Chen Y (2016) Roles of Circular RNAs in Neurologic Disease.

Front. Mol. Neurosci. 9:25.

doi: 10.3389/fnmol.2016.00025

\section{CircRNA STRUCTURE}

CircRNAs are a novel type of endogenous noncoding RNA, different from the linear RNAs, which form closed loop structures with a covalent bond linking the $3^{\prime}$ and $5^{\prime}$ ends. Consequently, they do not possess polyadenylated tails (Jeck et al., 2013; Chen et al., 2015). Classic RNA detection methods can only separate RNA molecules with polyadenylated tails, so circRNAs were ignored in previous studies. Since the development of high-throughput sequencing techniques and bioinformatics analysis programs, researchers have discovered thousands of circRNAs in mammalian cells (Salzman et al., 2012; Memczak et al., 2013). CircRNAs are abundant in plasma, sometimes at levels 10-folds higher than the corresponding linear mRNAs, due to their increased stability. Nuclease hydrolyzes can recognize the tails of linear RNAs, while circular RNAs form covalently closed loop structures that protect them. As a result, circular RNAs are not susceptible to degradation by RNA exonuclease or RNase (Salzman et al., 2012; Jeck et al., 2013; Memczak et al., 2013). CircRNAs are highly enriched in eukaryotic organisms and display elevated sequence conservation with specific expression in various tissues during different developmental stages. CircRNAs mainly arise from the exons of protein-coding genes and can also be derived from intronic areas, untranslated regions (UTRs), intergenic loci and antisense sequences of known transcripts (Jeck et al., 2013; Memczak et al., 2013). CircRNA biogenesis remains unclear, several models were proposed to explain the possible formation of ecircRNAs: Lariat, Intron-pairing, and resplicing-driven circularization (Figure 1; Chen et al., 2015). Some circRNAs may have miRNA response elements (MREs) and can interact with miRNAs (Hansen et al., 2011). 
A

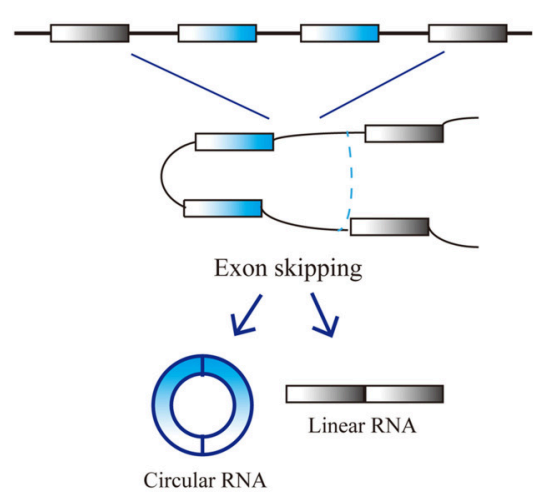

C

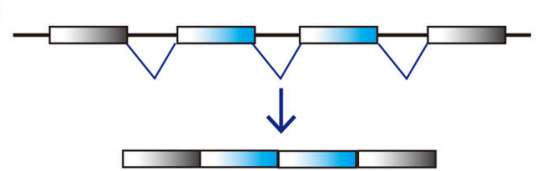

B

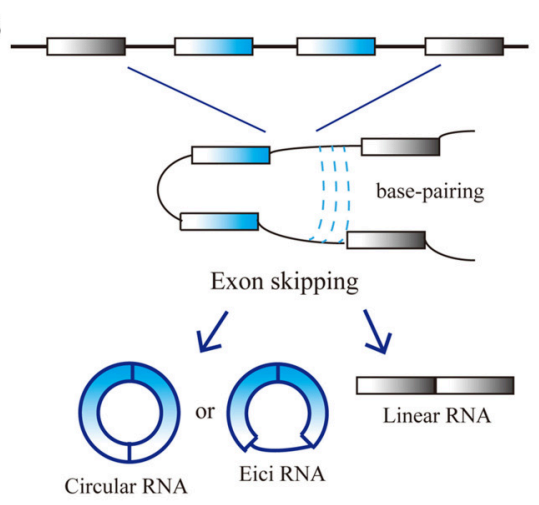

D

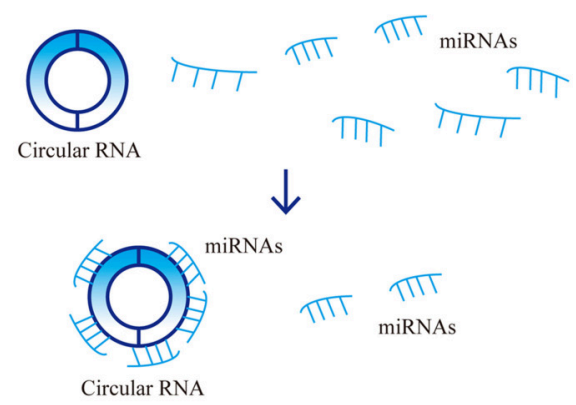

FIGURE 1 | Possible models and function of circRNA biogenesis. (A) Lariat-driven circularization, (B) Intron-pairing-driven circularization, (C) Resplicing-driven circularization, and (D) Function as miRNAs sponge.

\section{CircRNA FUNCTION}

Due to their high expression level and stability, circRNAs can act as competitive endogenous RNAs (ceRNAs). CeRNAs contain shared MREs and can compete for miRNA binding. Furthermore, circRNAs have the same selective capture of transcribed sequences with corresponding linear mRNAs, which may perform specific functions through influencing the combination of other RNAs. CircRNAs play an important role in the microadjustment of miRNA expression levels. CircRNAs can perturb miRNA function by competing with miRNA combination, thus preventing miRNA's effect on the posttranslational repression of target-coding RNA species, and then regulating the expression levels of target genes (Hansen et al., 2013a; Rybak-Wolf et al., 2015). Studies have revealed that the ability of circRNAs to combine with miRNAs is 10 times higher than other known transcriptions (Chen et al., 2015). A circRNA antisense to the cerebellar degeneration-related protein 1 transcript (CDR1as) can harbor more than $70 \mathrm{miR}-7$ binding sites and cannot be degraded by RNA-induced silencing complexes (RISCs) (Hansen et al., 2013a). Acting as a natural miRNA sponge, CDR1as can negatively regulate miR-7 (Salmena et al., 2011; Danan et al., 2012; Memczak et al., 2013). Therefore, CDR1as is also called the circular RNA sponge for miR-7 (CiRS-7). High CDRlas expression can decrease miR-7 activity by binding to it and thus increasing target gene expression; therefore, low CDR1as expression decreases miR-7 target gene expression. Moreover,
CDRlas seems to be degraded by miR-671 but not miR7 (Hansen et al., 2011, 2013a; Memczak et al., 2013). This indicates that circRNAs can transport miRNAs. CDR1as may first combine with miR-7 and transport it to specific sites, where miR-671 can degrade it and release miR-7. This characteristic is bound to affect ceRNA function because the combination and release of multiple miRNAs by circRNAs can affect hundreds of transcripts (Taulli et al., 2013). Studies have demonstrated that circRNA from sex-determining region $\mathrm{Y}$ (sry) harbors 16 miR-138 binding sites and can act as natural miRNA sponges to repress miR-138 activity (Hansen et al., 2013a). There are several other possible circRNA functions. First, they can regulate the expression of other RNAs through partial base pairing. For instance, CDR1as can bind with CDR1 mRNA based on complementary base pairing, thus increasing CDR1 mRNA stability (Hansen et al., 2011). Second, circRNA can combine with proteins and regulate their activity or recruit the components of multiprotein complexes (Bohjanen et al., 1996; Hansen et al., 2013a; Hentze and Preiss, 2013; Memczak et al., 2013). For example, CDR1as can bind with Argonaute (AGO) protein. Finally, circRNAs can act as translation templates to encode proteins (Chen and Sarnow, 1995; Perriman and Ares, 1998). CircRNAs may play significant roles in nervous system. Studies have found that CircRNAs are highly abundant in central nervous system and are differentially expressed in the brain. circRNAs are highly enriched in synapses and the expression level of circRNAs are differentially during neuronal differentiation (Rybak-Wolf 
et al., 2015). The above characteristics suggest that circRNAs may participate in development of nervous system, especially in synapse development and plasticity.

\section{CircRNA IN NEUROLOGICAL DISEASE}

Recent investigations have suggested that circRNAs may play crucial roles in the occurrence and development of neurological diseases and therefore have potential as novel biomarkers (Table 1). At present, few studies have directly assessed the functions and mechanisms of circRNAs in neurological diseases; rather, most are only related to circRNAs functions as miRNA regulators. One publication reported that circRNAs are extraordinarily enriched in the brain, especially in neuropils and dendrites, and may participate in regulating synaptic function and neural plasticity (You et al., 2015). These characteristics suggest that circRNAs could play important roles in nervous system diseases such as epilepsy, Parkinson's disease (PD), and Alzheimer's disease (AD). CDR1as overexpression in zebrafish embryos decreased midbrain size, which is the same effect observed following miR-7 silencing (Memczak et al., 2013). A large number of studies have demonstrated that miR-7 influences different signaling pathways by acting on other regulatory factors such as epidermal growth factor receptor (EGFR), insulin receptor substrate-1 (IRS-1), IRS-2, p21-activated kinase1 (Pak1), and Raf1 (Reddy et al., 2008; Webster et al., 2009). Therefore, CDRlas may be an important regulatory factor in the pathogenesis and progression of neurological diseases.

\section{CircRNA AND AD}

Functional deficiency of CDR1as can upregulate miR-7 expression and may lead to the downregulation of AD-relevant targets, such as ubiquitin protein ligase A (UBE2A; Bingol and Sheng, 2011; Lonskaya et al., 2013; Lukiw, 2013). This autophagic protein is important for clearing amyloid peptides and is depleted in the AD brain (Lonskaya et al., 2013). This suggests that CDR1as may participate in $\mathrm{AD}$ pathogenesis. In addition, circRNA from sry can act as a natural miRNA sponge to repress miR-138 activity (Hansen et al., 2013a). Some studies have shown miR-138 influences learning and memory abilities by regulating acyl protein thioesterase 1 (APT1; Tatro et al., 2013; Schröder et al., 2014).

\section{CircRNA AND PD}

As mentioned previously, CDR1as is a negative regulator of miR7 (Salmena et al., 2011; Danan et al., 2012; Memczak et al., 2013). Investigations have revealed that miR-7 can downregulate the expression of $\alpha$-synuclein, which is the main component of Lewy bodies in the PD brain. High neuronal expression of $\alpha$-synuclein protein is highly implicated in PD pathogenesis. Moreover, miR7 -induced $\alpha$-synuclein downregulation can also protect cells against oxidative stress (Junn et al., 2009). miRNA-7 can also protect against 1-methyl-4-phenylpyridinium-induced cell death by targeting the nuclear factor (NF) $-\kappa \mathrm{B}$ signaling pathway (Choi et al., 2014).

\section{CircRNA AND CEREBROVASCULAR DISEASE}

Circular antisense non-coding RNA in the INK4 locus (cANRIL) is an antisense transcript from the cyclin-dependent kinase 4 inhibitor (CDK4a, also called INK4a) alternative reading frame locus (Salzman et al., 2013). Single nucleotide polymorphisms (SNPs) near INK4/ARF are associated with atherosclerotic vascular disease. The cANRIL locus is within the SNPs and the nearest coding gene, and CANRIL can influence INK4/ARF expression and increase the risk of ASVD (Burd et al., 2010).

\begin{tabular}{|c|c|c|c|}
\hline Neurological disease & Circular RNA & Targets & Mechanisms \\
\hline \multirow[t]{2}{*}{ AD } & CDR1as & $\operatorname{miR}-7$ & $\begin{array}{l}\text { miR-7 can down-regulate AD-relevant targets, such as UBE2A, which play an important role in the } \\
\text { clearance of amyloid peptides in AD (Bingol and Sheng, 2011; Lukiw, 2013) }\end{array}$ \\
\hline & Sry & $\operatorname{miR}-138$ & miR-138 participate in learning and memory ability by regulating APT1 (Hansen et al., 2013a) \\
\hline PD & CDR1as & $\operatorname{miR}-7$ & $\begin{array}{l}\text { miR-7 can downregulate } \alpha \text {-synuclein expression and protect cells against oxidative stress (Junn } \\
\text { et al., 2009) }\end{array}$ \\
\hline Cerebrovascular disease & CANRIL & INK4a & cANRIL can influence INK4/ARF expression and increase the risk of ASVD (Salzman et al., 2013) \\
\hline \multirow[t]{3}{*}{ Inflammatory neuropathy } & hsa-circRNA 2149 & & Specific expression in leukocytes \\
\hline & CircRNA100783 & & CircRNA100783 may be involved in chronic CD28-associated CD8(+)T cell aging (Wang et al., 2015) \\
\hline & Sry & $\operatorname{miR}-138$ & $\begin{array}{l}\text { miR-138 can balance the expression between Th1 and Th2 through suppressing the function of } \\
\text { RUNX3 (Fu et al., 2015) }\end{array}$ \\
\hline Nervous System Neoplasms & CDR1as & miR-7 & $\begin{array}{l}\text { miR-7 can repress the expression of EGFR, IRS-1 and IRS-2 thus reduce the active and aggressive } \\
\text { of glioblastoma (Liu et al., 2014) }\end{array}$ \\
\hline
\end{tabular}


This observation indicates that circRNA may be involved in the development of atherosclerotic cerebrovascular disease.

\section{CircRNA AND INFLAMMATORY NEUROPATHY}

Some circRNAs seem to have virus miRNA binding sites and therefore can affect immune responses. For example, hsacircRNA 2149 contains 13 unique, head-to-tail spanning reads. Researchers detected hsa-circRNA 2149 in CD19+ leukocytes but not CD341 leukocytes, neutrophils, or HEK293 cells. Besides, circular RNA100783 may be involved in chronic CD28associated CD8(+)T cell aging and could therefore be a novel biomarker for this conditions (Wang et al., 2015). CircRNA from sry can repress miR-138 activity. Studies found that miR-138 could balance T helper 1 (Th1) and T helper 2 (Th2) expressions through suppressing the function of runt-related transcription factor 3 (RUNX3; Fu et al., 2015). The above studies suggest that circRNA may participate in inflammatory reactions that induce neuropathy.

\section{CircRNA AND NERVOUS SYSTEM NEOPLASMS}

Several circRNAs play critical roles in cancer related biological processes and are dysregulated in cancer tissues ( $\mathrm{Li}$ et al., 2015a,b; Qin et al., 2016). CDR1as was first found in brain tissue and is widely expressed in the nervous system, especially the cerebrum. Expression analyses of various tumor cell lines showed widespread expression of CDR1as in neuroblastomas and astrocytoma (Dropcho et al., 1987; Chen et al., 1990; Hansen et al., 2013b). Researchers measured miRNA expressions in different tumor cell lines and found that compared to normal brain tissue, miR-7 was down-regulated in astrocytoma and neuroblastoma, Research on a glioblastoma cell line demonstrated that miR-7 could repress EGFR expression and also downregulate IRS- 1 and IRS- 2 expression by suppressing the activity of protein kinase B (PKB; Liu et al., 2014). CDRlas acts as negative regulator of miR-7 (Hansen et al., 2013a). These lines of evidence indicate that circRNAs may be

\section{REFERENCES}

Arnberg, A. C., Van Ommen, G. J., Grivell, L. A., Van Bruggen, E. F., and Borst, P. (1980). Some yeast mitochondrial RNAs are circular. Cell 19, 313-319. doi: 10.1016/0092-8674(80)90505-X

Bingol, B., and Sheng, M. (2011). Deconstruction for reconstruction: the role of proteolysis in neural plasticity and disease. Neuron 69, 22-32. doi: 10.1016/j.neuron.2010.11.006

Bohjanen, P. R., Colvin, R. A., Puttaraju, M., Been, M. D., and Garcia-Blanco, M. A. (1996). A small circular TAR RNA decoy specifically inhibits Tat-activated HIV1 transcription. Nucleic Acids Res. 24, 3733-3738. doi: 10.1093/nar/24.19.3733

Burd, C. E., Jeck, W. R., Liu, Y., Sanoff, H. K., Wang, Z., and Sharpless, N. E. (2010). Expression of linear and novel circular forms of an INK4/ARF-associated noncoding RNA correlates with atherosclerosis risk. PLoS Genet. 6:e1001233. doi: 10.1371/journal.pgen.1001233 involved in the pathogenesis and progression of nervous system neoplasms.

\section{CircRNA AND PRION DISEASES}

Prion diseases are devastating neurodegenerative disorders including Creutzfeldt-Jakob disease (CJD), kuru, GerstmannStraussler syndrome, and fatal familial insomnia (FFI; Prusiner, 1998). Most prion diseases are infectious via transmissible particles composed of scrapie prion protein (PrPSc), an isomer of cellular prion protein (PrPC). Studies have revealed that CDRlas expression is induced by PrPC overexpression (Satoh and Yamamura, 2004; Satoh et al., 2009). Therefore, CDR1as may be involved in the prion disease pathogenesis.

\section{CONCLUSIONS}

CircRNA function and their relationships with neurological diseases remain to be fully elucidated, and some problems remainto be solved. Due to the abundance and stability of circRNAs in vivo, they may be useful clinical diagnosis biomarkers in the future. Meanwhile, the regulatory effects of circRNAs on genes may be considered as treatment targets. In addition, the ability of circRNAs to act as sponges of related miRNAs can be exploited as a novel technology to achieve gene regulation, different cytotypes could differentially be regulated with a selective silencing approach. Further study on circRNA structure and function will improve our understanding regarding neurological disease pathogenesis and lead to new diagnostic and treatment methods.

\section{AUTHOR CONTRIBUTIONS}

YS conceived the content and wrote the critical review. YC provided the ideas and supervised the work. Both authors read and approved the final version of the manuscript.

\section{ACKNOWLEDGMENTS}

This work was supported through grants from the National Natural Science Foundation of China (81571261) and the Shanghai Committee of Science and Technology (14411972500).

Chen, C. Y., and Sarnow, P. (1995). Initiation of protein synthesis by the eukaryotic translational apparatus on circular RNAs. Science 268, 415-417. doi: 10.1126/science.7536344

Chen, I., Chen, C. Y., and Chuang, T. J. (2015). Biogenesis, identification, and function of exonic circular RNAs. Wiley Interdiscip. Rev. RNA 6, 563-579. doi: 10.1002/wrna.1294

Chen, Y. T, Rettig, W. J., Yenamandra, A. K., Kozak, C. A., Chaganti, R. S., and Posner, J. B., et al. (1990). Cerebellar degeneration-related antigen: a highly conserved neuroectodermal marker mapped to chromosomes $\mathrm{X}$ in human and mouse. Proc. Natl. Acad. Sci. U.S.A. 87, 3077-3081. doi: 10.1073/pnas.87. 8.3077

Choi, D. C., Chae, Y. J., Kabaria, S., Chaudhuri, A. D., Jain, M. R., Li, H., et al. (2014). MicroRNA-7 protects against 1-methyl-4-phenylpyridiniuminduced cell death by targeting RelA. J. Neurosci. 34, 12725-12737. doi: 10.1523/JNEUROSCI.0985-14.2014 
Cocquerelle, C., Mascrez, B., Hétuin, D., and Bailleul, B. (1993). Mis-splicing yields circular RNA molecules. FASEB J. 7, 155-160.

Danan, M., Schwartz, S., Edelheit, S., and Soreak, R. (2012). Transcriptome-wide discovery of circular RNAs in Archaea. Nucleic Acids Res. 40, 3131-3142. doi: 10.1093/nar/gkr1009

Dropcho, E. J., Chen, Y. T., Posner, J. B., and Old, L. J. (1987). Cloning of a brain protein identified by autoantibodies from a patient with paraneoplastic cerebellar degeneration. Proc. Natl. Acad. Sci. U.S.A. 84, 4552-4556. doi: 10.1073/pnas.84.13.4552

Fu, D., Yu, W., Li, M., Wang, H., Liu, D., Song, X., et al. (2015). MicroRNA-138 regulates the balance of Th1/Th2 via targeting RUNX3 in psoriasis. Immunol Lett. 166, 55-62. doi: 10.1016/j.imlet.2015.05.014

Hansen, T. B., Jensen, T. I., Clausen, B. H., Bramsen, J. B., Finsen, B., Damgaard, C. K., et al. (2013a). Natural RNA circles function as efficient microRNA sponges. Nature 495, 384-388. doi: 10.1038/nature11993

Hansen, T. B., Kjems, J., and Damgaard, C. K. (2013b). Circular RNA and miR-7 in cancer. Cancer Res. 73, 5609-5612. doi: 10.1158/0008-5472.CAN13-1568

Hansen, T. B., Wiklund, E. D., Bramsen, J. B., Villadsen, S. B., Statham, A. L., Clark, S. J., et al. (2011). miRNA-dependent gene silencing involving Ago2mediated cleavage of a circular antisense RNA. EMBO J. 30, 4414-4422. doi: 10.1038/emboj.2011.359

Hentze, M. W., and Preiss, T. (2013). Circular RNAs: splicing's enigma variations. EMBO J. 32, 923-925. doi: 10.1038/emboj.2013.53

Jeck, W. R., Sorrentino, J. A., Wang, K., Slevin, M. K., Burd, C. E., Liu, J., et al. (2013). Circular RNAs are abundant, conserved, and associated with ALU repeats. RNA 19, 141-157. doi: 10.1261/rna.035667.112

Junn, E., Lee, K. W., Jeong, B. S., Chan, T. W., Im, J. Y., and Mouradian, M. M. (2009). Repression of alpha-synuclein expression and toxicity by microRNA7. Proc. Natl. Acad. Sci. U.S.A. 106, 13052-13057. doi: 10.1073/pnas.0906 277106

Li, F., Zhang, L., Li, W., Deng, J., Zheng, J., An, M., et al. (2015b). Circular RNA ITCH has inhibitory effect on ESCC by suppressing the Wnt/beta-catenin pathway. Oncotarget 6, 6001-6013. doi: 10.18632/oncotarget.3469

Li, Y., Zheng, Q., Bao, C., Li, S., Guo, W., Zhao, J., et al. (2015a). Circular RNA is enriched and stable in exosomes: a promising biomarker for cancer diagnosis. Cell Res. 25, 981-984. doi: 10.1038/cr.2015.82

Liu, Z., Jiang, Z., Huang, J., Huang, S., Li, Y., Yu, S., et al. (2014). miR7 inhibits glioblastoma growth by simultaneously interfering with the PI3K/ATK and Raf/MEK/ERK pathways. Int. J. Oncol. 44, 1571-1580. doi: $10.3892 /$ ijo.2014.2322

Lonskaya, I., Shekoyan, A. R., Hebron, M. L., Desforges, N., Algarzae, N. K., and Moussa, C. E. (2013). Diminished parkin solubility and co-localization with intraneuronal amyloid-beta are associated with autophagic defects in Alzheimer's disease. J. Alzheimers Dis. 33, 231-247. doi: 10.3233/JAD-2012121141

Lukiw, W. J. (2013). Circular RNA (circRNA) in Alzheimer's disease (AD). Front. Genet. 4:307. doi: 10.3389/fgene.2013.00307

Memczak, S., Jens, M., Elefsinioti, A., Torti, F., Krueger, J., Rybak, A., et al. (2013). Circular RNAs are a large class of animal RNAs with regulatory potency. Nature 495, 333-338. doi: 10.1038/nature11928

Perriman, R., and Ares, M. J. (1998). Circular mRNA can direct translation of extremely long repeating-sequence proteins in vivo. RNA 4, 1047-1054. doi: 10.1017/S135583829898061X

Prusiner, S. B. (1998). Prions. Proc. Natl. Acad. Sci. U.S.A. 95, 13363-13383. doi: 10.1073/pnas.95.23.13363

Qin, M., Liu, G., Huo, X., Tao, X., Sun, X., Ge, Z., et al. (2016). Hsa_circ_0001649: a circular RNA and potential novel biomarker for hepatocellular carcinoma. Cancer Biomark 16, 161-169. doi: 10.3233/CBM-150552
Reddy, S. D., Ohshiro, K., Rayala, S. K., and Kumar, R. (2008). MicroRNA7, a homeobox D10 target, inhibits p21-activated kinase 1 and regulates its functions. Cancer Res. 68, 8195-8200. doi: 10.1158/0008-5472.CAN-08-2103

Rybak-Wolf, A., Stottmeister, C., Glažar, P., Jens, M., Pino, N., Giusti, S., et al. (2015). Circular RNAs in the mammalian brain are highly abundant, conserved, and dynamically expressed. Mol Cell 58, 870-885. doi: 10.1016/j.molcel.2015.03.027

Salmena, L., Poliseno, L., Tay, Y., Kats, L., and Pandolfi, P. P. (2011). A ceRNA hypothesis: the rosetta stone of a hidden RNA language? Cell 146, 353-358. doi: 10.1016/j.cell.2011.07.014

Salzman, J., Chen, R. E., Olsen, M. N., Wang, P. L., and Brown, P. O. (2013). Celltype specific features of circular RNA expression. PLoS Genet. 9:e1003777. doi: 10.1371/journal.pgen.1003777

Salzman, J., Gawad, C., Wang, P. L., Lacayo, N., and Brown, P. O. (2012). Circular RNAs are the predominant transcript isoform from hundreds of human genes in diverse cell types. PLoS ONE 7:e30733. doi: 10.1371/journal.pone.0030733

Sanger, H. L., Klotz, G., Riesner, D., Gross, H. J., and Kleinschmidt, A. K. (1976). Viroids are single-stranded covalently closed circular RNA molecules existing as highly base-paired rod-like structures. Proc. Natl. Acad. Sci. U.S.A. 73, 3852-3856. doi: 10.1073/pnas.73.11.3852

Satoh, J., Obayashi, S., Misawa, T., Sumiyoshi, K., Oosumi, K., and Tabunoki, H. (2009). Protein microarray analysis identifies human cellular prion protein interactors. Neuropathol. Appl. Neurobiol. 35, 16-35. doi: 10.1111/j.13652990.2008.00947.x

Satoh, J., and Yamamura, T. (2004). Gene expression profile following stable expression of the cellular prion protein. Cell. Mol. Neurobiol. 24, 793-814. doi: 10.1007/s10571-004-6920-0

Schröder, J., Ansaloni, S., Schilling, M., Liu, T., Radke, J., Jaedicke, M., et al. (2014). MicroRNA-138 is a potential regulator of memory performance in humans. Front. Hum. Neurosci. 8:501. doi: 10.3389/fnhum.2014.00501

Tatro, E. T., Risbrough, V., Soontornniyomkij, B., Young, J., ShumakerArmstrong, S., Jeste, D. V., et al. (2013). Short-term recognition memory correlates with regional CNS expression of microRNA-138 in mice. Am. J. Geriatr. Psychiatry 21, 461-473. doi: 10.1016/j.jagp.2012.09.005

Taulli, R., Loretelli, C., and Pandolfi, P. P. (2013). From pseudo-ceRNAs to circceRNAs: a tale of cross-talk and competition. Nat. Struct. Mol. Biol. 20, 541-543. doi: $10.1038 / \mathrm{nsmb} .2580$

Wang, Y. H., Yu, X. H., Luo, S. S., and Han, H. (2015). Comprehensive circular RNA profiling reveals that circular RNA100783 is involved in chronic CD28associated CD8(+)T cell ageing. Immun. Ageing 12, 17. doi: 10.1186/s12979015-0042-z

Webster, R. J., Giles, K. M., Price, K. J., Zhang, P. M., Mattick, J. S., and Leedman, P. J. (2009). Regulation of epidermal growth factor receptor signaling in human cancer cells by microRNA-7. J. Biol. Chem. 284, 5731-5741. doi: 10.1074/jbc.M804280200

You, X., Vlatkovic, I., Babic, A., Will, T., Epstein, I., Tushev, G., et al. (2015). Neural circular RNAs are derived from synaptic genes and regulated by development and plasticity. Nat. Neurosci. 18, 603-610. doi: 10.1038/nn.3975

Conflict of Interest Statement: The authors declare that the research was conducted in the absence of any commercial or financial relationships that could be construed as a potential conflict of interest.

Copyright $\odot 2016$ Shao and Chen. This is an open-access article distributed under the terms of the Creative Commons Attribution License (CC BY). The use, distribution or reproduction in other forums is permitted, provided the original author(s) or licensor are credited and that the original publication in this journal is cited, in accordance with accepted academic practice. No use, distribution or reproduction is permitted which does not comply with these terms. 